Conclusion Geosexually isolated participants demonstrated "convection mixing", with repeated movement from peripheral, to downtown, back to peripheral areas. More attention should be paid to identifying STI reservoirs outside of core areas.

Disclosure No significant relationships.

\section{P516 LOW IMMUNITY TO HEPATITIS A AMONGST MEN WHO HAVE SEX WITH MEN ATTENDING A LARGE SEXUAL HEALTH CLINIC IN MELBOURNE, AUSTRALIA}

Lenka Vodstrcil*, Christopher Fairley, Catriona Bradshaw, Marcus Chen, Eric Chow. Monash University, Central Clinical School, Carlton, Australia

10.1136/sextrans-2019-sti.594

Background Recent outbreaks of hepatitis A have been reported globally, and increasing numbers of cases and deaths are being reported in Australia, particularly among men who have sex with men (MSM). The critical vaccination threshold for hepatitis A has been estimated to be $\geq 70 \%$ to prevent outbreaks in MSM. This study aimed to determine the level of immunity to hepatitis A among MSM since 2012.

Methods This was a retrospective audit of serological testing data from first-time MSM attendees at the Melbourne Sexual Health Centre (MSHC) from 2012-2018. We determined the proportion of MSM who were tested and who had serological detection of hepatitis A antibodies, stratified by age and year. We used logistic regression to investigate factors associated with detection of hepatitis A antibodies.

Results There were 16,615 new MSM attendees at MSHC over the 7-year period, of which 9,719 (58\%, 95\% CI:57,59\%) were tested for hepatitis A antibodies. There was a $2 \%$ annual increase in the proportion of men tested over time (from 60\% in 2012 to $69 \%$ in $2018 ; \mathrm{p}=0.025$ ). Of those tested, 44\% ( $n=4,304,95 \% \mathrm{CI}: 43,45 \%)$ of men had hepatitis A antibodies detected at their first visit, but there was no change over time $(p=0.201)$. However, compared to our last audit that spanned 2002-2011, a higher proportion of young MSM (aged $<20$ years) were tested for hepatitis A at their first visit (73\% vs $33 \%$ before 2012) and had antibodies detected (38\% vs 19\% before 2012). Detection of hepatitis A antibodies was associated with age $\geq 30$ (Adjusted $\mathrm{OR}=1.89$, 95\%CI:1.77,2.02), being born overseas vs Australia/New Zealand $(\mathrm{AOR}=1.23,95 \% \mathrm{CI}: 1.13,1.33)$, and consistent condom use in the last 12 months (AOR=1.19, 95\%CI:1.12,1.28).

Conclusion Hepatitis A immunity amongst MSM remains far below the estimated $70 \%$ required to prevent outbreaks. Measures including increased testing and higher vaccination coverage are needed to limit the number of cases and deaths. Disclosure No significant relationships.

\section{P517 DO SEXUAL PRACTICES DIFFER BY AGE AMONG GAY AND BISEXUAL MEN? A CROSS-SECTIONAL STUDY IN MELBOURNE, AUSTRALIA}

Alex Kilner, Christopher Fairley, Sam Burrell, Catriona Bradshaw, Marcus Chen, Eric Chow* Alfred Health, Melbourne Sexual Health Centre, Carlton, Australia

10.1136/sextrans-2019-sti.595
Background Unlike chlamydia, gonorrhoea is considerably more common in young men who have sex with men (MSM). This may relate to differences in sexual practices although currently studies predominantly focus on anal sex with very limited data on fellatio and kissing. This study aimed to examine a range of different sexual practices among MSM.

Methods MSM who attended the Melbourne Sexual Health Clinic in 2017 were invited to participate in a survey on nine sexual practices engagement with their most recent regular and/or casual partner(s). This included: kissing, touching penises, mutual masturbation, giving/receiving fellatio, giving/ receiving rimming as well as insertive/receptive anal sex. Chisquared trend test was used to examine the age patterns of different sexual practice.

Results 1601 men were included with a median age of 30 (IQR 25-36). Kissing was the most common practice among casual partners (92.4\%), followed by giving fellatio $(85.8 \%)$ and receiving fellatio $(83.9 \%)$. The least common activity was giving rimming (38.0\%), followed by receiving rimming $(45.8 \%)$. The proportion of kissing decreased with increasing age $(95.0 \%$ among men aged $<30$ vs $79.1 \%$ among men aged $\left.\geq 50, \mathrm{p}_{\text {trend }}<0.001\right)$. Receiving rimming also decreased with increasing age $\left(p_{\text {trend }}=0.034\right)$. Receptive anal sex was more common among younger men ( $\mathrm{p}_{\text {trend }}<$ 0.001 ) but insertive anal sex was more common among older men than younger men $\left(p_{\text {trend }}=0.002\right)$. Sexual activity had fewer correlations with age among regular partners. Younger men were more likely to masturbate mutually $\left(p_{\text {trend }}=0.031\right)$ and receive anal sex from their regular partners $\left(p_{\text {trend }}=0.013\right.$ ).

Conclusion Age is strongly associated with sexual practices among casual partners but less so among regular partners. Younger men more likely to engage in activities associated with gonorrhoea transmission such as kissing and receptive rimming.

Disclosure No significant relationships.

\section{P518 SEXUAL PRACTICES AND HEALTHCARE USE OF MEN WHO HAVE SEX WITH MEN ONLY AND MEN WHO HAVE SEX WITH MEN AND WOMEN}

${ }^{1}$ Tom Smardon, ${ }^{1}$ Alaina Vaisey*, ${ }^{2}$ Eric Chow, ${ }^{3}$ Christopher Fairley, ${ }^{4}$ Jane Hocking. ${ }^{1}$ University of Melbourne, Melbourne School of Population and Global Health, Carlton, Australia; ${ }^{2}$ Alfred Health, Melbourne Sexual Health Centre, Carlton, Australia; ${ }^{3}$ Melbourne Sexual Health Centre, Melbourne, Australia; ${ }^{4}$ University of Melbourne, Melbourne School of Population and Global Health, Parkville, Australia

\subsection{6/sextrans-2019-sti.596}

Background It is unclear why gonorrhoea and syphilis rates are increasing among women in Australia. One possible reason is transmission to women from men who have sex with men and women (MSMW). We aimed to explore sexual practices and healthcare use of MSMW and men who have sex with men only (MSMO).

Methods Semi-structured interviews were conducted. Participants were recruited from a public sexual health clinic and via community advertisements in Melbourne, Australia. Men were eligible if they were cisgender, aged $18+$ years and had sex with either men or men and women in the last 12 months. Data were analysed thematically. 
Results 28 interviews were conducted (15 MSMO, 13 MSMW). Half of MSMW reported predominantly male partners and half predominantly female. MSMO viewed STIs as "an annoying reality of life" and both groups had positive perceptions of STI testing; however, MSMW described more STI stigma and less frequent testing. MSMO and MSMW who were more involved with the queer community had better sexual health knowledge. Many MSMW noted it was easier to have sexual health discussions with male partners; however, many also described the pressure of condom use, "with men, [was] to not use a condom a fair bit of time and probably by women, [was] to use a condom." There was significant fear in both groups about disclosing sexual practices to general practitioners and some MSMW preferred the anonymity of specialist sexual health clinics. Biphobia and bisexual erasure were frequently discussed by both groups.

Conclusion MSMW described less comprehensive sexual health knowledge and more barriers to accessing sexual health care. Service provision and health promotion messaging must be broadened to capture the reality of increasing sexual fluidity. Destigmatising MSM behaviour and sexual health discussions, particularly in primary care, is crucial to ensuring all people receive appropriate sexual health care.

Disclosure No significant relationships.

\section{P519 IS CHEMSEX AMONG MEN WHO HAVE SEX WITH MEN PERCEIVED AS PROBLEMATIC? A CROSS-SECTIONAL STUDY IN THE NETHERLANDS}

\begin{abstract}
${ }^{1}$ Ymke Evers, ${ }^{1}$ Christian Hoebe*, ${ }^{1}$ Nicole Dukers-Muijers, ${ }^{2}$ Karlijn Kampman, ${ }^{3}$ Sophie Kuizenga, ${ }^{4}$ Decontee Shilue, ${ }^{5}$ Nienke Bakker, ${ }^{6}$ Sophie Schamp, ${ }^{1}$ Geneviève Van Liere. 'Public Health Service South Limburg, Maastricht University Medical Center (MUMC), Sexual Health, Infectious Diseases and Environmental Health, Medical Microbiology, Care and Public Health Research Institute (CAPHRI), Heerlen, Netherlands; ${ }^{2}$ Public Health Service Twente, Sexual Health, Twente, Netherlands; ${ }^{3}$ Public Health Service Haaglanden, Sexual Health, Den Haag, Netherlands; ${ }^{4}$ Public Health Service Middle Brabant, Sexual Health, Middle Brabant, Netherlands; ${ }^{5}$ Public Health Service Middle Gelderland, Sexual Health, Middle Gelderland, Netherlands; ${ }^{6}$ Public Health Service Brabant South-East, Sexual Health, Brabant South-East, Netherlands
\end{abstract}

10.1136/sextrans-2019-sti.597

Background Men who have sex with men (MSM) are increasingly using drugs during sex ('chemsex') and this has been associated with several health harms, including an increased risk for sexually transmitted infections (STI) and addiction. Little evidence exists on whether chemsex is perceived as problematic by MSM. This study assessed a wide range of social and behavioral aspects in MSM engaging in chemsex.

Methods In 2018, 785 MSM were recruited at eight Dutch STI clinics, of which $511(65 \%)$ completed the online questionnaire. Chemsex was defined as using cocaine, crystal meth, designer drugs, GHB/GBL, ketamine, speed or XTC/MDMA during sex in the preceding six months. Characteristics of MSM intending to change chemsex behaviour and MSM with a need for professional counselling were described using $\chi^{2}$ tests.

Results Chemsex was reported by 41\% (209/511). Among MSM engaging in chemsex, intention to change was reported by $19 \%$ (40/209); decreasing the frequency of drug use was mostly reported $(70 \%(28 / 40))$. Intention to change was highest among MSM who had no sex without drugs $<3$ months $(44 \%(12 / 47)$ vs $15 \%, \mathrm{p}<0.001)$, reported unwanted sexual experiences $(36 \%(14 / 39)$ vs $15 \%, \quad \mathrm{p}=0.01)$, and used $\geq 5$ drugs $<6$ months $(31 \%(16 / 51)$ vs $16 \%, \mathrm{p}=0.03)$. The need for professional counselling was reported by $23 \%$ (48/209). The majority wanted to be counselled on increasing self-control $(52 \%(25 / 48))$. The need for professional counselling was highest among MSM who had an intention to change $(45 \%$ $(18 / 40)$ vs $18 \%, p<0.001)$, had no sex without drugs $<3$ months $(41 \%(11 / 27)$ vs $20 \%, p=0.04)$, and engaged in chemsex $\geq 2$ times per month $(30 \%(28 / 93)$ vs $17 \%, \mathrm{p}=0.03)$.

Conclusion Our study shows that one in five MSM engaging in chemsex reported an intention to change or expressed a need for professional counselling. STI healthcare providers should discuss chemsex frequency, multiple drug use, sober sex and unwanted sexual experiences, and if necessary refer to professional addiction-or mental healthcare.

Disclosure No significant relationships.

\section{P520 HPV INFECTIONS AND FLAT PENILE LESIONS OF THE PENIS IN MEN WHO HAVE SEX WITH MEN}

${ }^{1}$ Ward Van Bilsen*, ${ }^{1}$ Alexandra Kovaleva, ${ }^{2}$ Maaike Bleeker, ${ }^{3}$ Audrey King, ${ }^{1}$ Sylvia Bruisten, ${ }^{4}$ Wilma Brokking, ${ }^{5}$ Henry De Vries, ${ }^{2}$ Chris Meijer, ${ }^{1}$ Maarten Schim Van Der Loeff ${ }^{*} .{ }^{1}$ Public Health Service of Amsterdam, Department of Infectious Diseases, Amsterdam, Netherlands; ${ }^{2}$ Amsterdam UMC, Vrije Universiteit-University Medical Center, Department of Pathology, Amsterdam, Netherlands; ${ }^{3}$ Center for Infectious Disease Control, National Institute for Public Health and the Environment (RIVM), Bilthoven, Netherlands; ${ }^{4} D C$ Klinieken, Department of Internal Medicine, Amsterdam, Netherlands; ${ }^{5}$ Public Health Service Amsterdam, Amsterdam University Medical Center (UMC), National Institute of Public Health and the Environment (RIVM), Infectious Diseases, Infection and Immunity Institute (AI and II), Epidemiology and Surveillance Unit, Amsterdam, Netherlands

\subsection{6/sextrans-2019-sti.598}

Background Flat penile lesions (FPL) in heterosexual men are thought to play a role in the transmission of HPV. We investigated the association between FPL and penile HPV, and explored determinants of FPL in men who have sex with men (MSM).

Methods In 2015-2016, MSM were recruited based on HIV and penile HPV status in a previous study. MSM self-completed a questionnaire. Peniscopy was performed after application of acetic acid to visualize FPL. Penile physician-collected samples were tested for HPV-DNA using the highly sensitive SPF10-PCR DEIA/LiPA25 system. If tested positive for HPV $6,11,16,18,31,33,35,39,45,51,52,56,58$ and/or 59, we determined the HPV viral load (VL), using type-specific L1-targeting quantitative (q)PCR. Presence of HPV and HIV, HPV VL and circumcision status were compared between MSM with and without FPL.

Results We included 116 MSM, of whom 59/116 (51\%) were HIV-positive and 54/116 (47\%) had FPL. A penile HPV infection was present in 31/54 (57\%) MSM with FPL and in 34/ $62(55 \%)$ MSM without FPL $(\mathrm{p}=0.8)$. Among MSM with FPL, 16/54 (30\%) had an hrHPV infection and 23/54 (43\%) had a lrHPV infection, which did not significantly differ from MSM without FPL $(p=0.5$ and $p=0.4$, respectively). A detectable HPV VL was found in 10/54 (19\%) MSM with FPL and in 10/62 $(16 \%)$ MSM without FPL $(p=0.6)$. Among MSM with FPL, $27 / 54$ (50\%) were HIV-positive and 5/54 (9\%) were circumcised, and among MSM without FPL, 32/62 (54\%) were HIV-positive and 13/62 (21\%) were circumcised $(\mathrm{p}=0.9$ and $\mathrm{p}=0.09$, respectively).

Conclusion Among MSM in Amsterdam, we found no association between FPL and penile HPV, HPV VL, HIV status or circumcision status, which is in contrast with findings among 